\title{
Treatment of Refractory Hypertension with Timely Angioplasty in Total Renal Artery Occlusion with Atrophic Kidney
}

\author{
Yuri Sasaki ${ }^{1}$, Eikan Mishima ${ }^{2}$, Koichi Kikuchi ${ }^{2}$, Takafumi Toyohara ${ }^{2}$, Takehiro Suzuki ${ }^{2}$, \\ Hideki Ota $^{3}$, Kazumasa Seiji ${ }^{3}$, Mariko Miyazaki ${ }^{2}$, Hideo Harigae ${ }^{2,4}$, Sadayoshi Ito ${ }^{2,5}$, \\ Kei Takase ${ }^{3}$ and Takaaki Abe ${ }^{2,6,7}$
}

\begin{abstract}
:
Angioplasty for cases of chronic total occlusion of renal artery with/without atrophic kidney is generally not recommended. We herein report a 57-year-old man who presented with renin-mediated refractory hypertension caused by occlusion of a unilateral renal artery leading to kidney atrophy (length: $69 \mathrm{~mm}$ ). Angioplasty favorably achieved blood pressure control with normalized renin secretion and enlargement of the atrophic kidney to $85 \mathrm{~mm}$. Timely angioplasty can be beneficial in select patients, even with an atrophic kidney and total occlusion, especially in cases with deterioration of hypertension within six months and the presence of collateral perfusion to the affected kidney.
\end{abstract}

Key words: renal artery stenosis, renovascular hypertension, renin-angiotensin system inhibitors, atherosclerotic renovascular disease, diffusion-weighted MRI

(Intern Med 60: 287-292, 2021)

(DOI: 10.2169/internalmedicine.5290-20)

\section{Introduction}

Atherosclerotic renovascular disease (ARVD) is the most common form of renal artery disease and is associated with severe comorbidities, such as renovascular hypertension with refractory hypertension and ischemic nephropathy with a progressive decline in the renal function (1). Percutaneous transluminal renal angioplasty (PTRA) with stenting is considered an effective modality for the correction of occlusive lesions and the subsequent normalization of the reduced renal blood flow. Nonetheless, randomized controlled trials evaluating angioplasty for ARVD have not found any advantages of angioplasty over medical therapy alone in terms of renal protection $(2,3)$. Based on these negative studies, guidelines no longer support routine angioplasty for ARVD patients in general (4-6). However, observational evidence indicating the benefits of PTRA in specific high-risk patients has been increasing (7-10). Thus, the correction of occlusive lesions through angioplasty has a beneficial effect in carefully selected patients who will potentially respond well to angioplasty.

Considering the indication for angioplasty, the current expert consensus statement recommends angioplasty as a therapeutic option for patients with developing ischemic nephropathy or high-risk manifestations of renovascular hypertension (11). However, cases with advanced atrophic kidney (pole-to-pole length $<7 \mathrm{~cm}$ ) and kidney with total occlusion of the renal artery have rarely been considered appropriate due to the limited benefits of angioplasty $(11,12)$.

${ }^{1}$ Tohoku University School of Medicine, Japan, ${ }^{2}$ Division of Nephrology, Endocrinology, and Vascular Medicine, Tohoku University Graduate School of Medicine, Japan, ${ }^{3}$ Department of Diagnostic Radiology, Tohoku University Graduate School of Medicine, Japan, ${ }^{4}$ Department of Hematology and Rheumatology, Tohoku University Graduate School of Medicine, Japan, ${ }^{5}$ Department of Medicine, Katta Public General Hospital, Japan, ${ }^{6}$ Division of Medical Science, Tohoku University Graduate School of Biomedical Engineering, Japan and ${ }^{7}$ Department of Clinical Biology and Hormonal Regulation, Tohoku University Graduate School of Medicine, Japan

Received: May 11, 2020; Accepted: July 6, 2020; Advance Publication by J-STAGE: August 22, 2020

Correspondence to Dr. Takaaki Abe, takaabe@med.tohoku.ac.jp 
We herein report a case of ARVD presenting with a sudden deterioration of refractory hypertension and atrophic kidney (length $<7 \mathrm{~cm}$ ) caused by total unilateral renal artery occlusion. In this case, timely revascularization of the occlusion by PTRA favorably achieved blood pressure control and enlargement of the atrophic kidney.

\section{Case Report}

A 57-year-old man had hypertension that had been diag-

Table. Laboratory Results of the Patient before PTRA.

\begin{tabular}{llc}
\hline White blood count & $(/ \mu \mathrm{L})$ & 8,300 \\
Hemoglobin & $(\mathrm{g} / \mathrm{dL})$ & 12.6 \\
Platelet count & $(/ \mu \mathrm{L})$ & $26.2 \times 10^{4}$ \\
Blood urea nitrogen & $(\mathrm{mg} / \mathrm{dL})$ & 16 \\
Creatinine & $(\mathrm{mg} / \mathrm{dL})$ & 1.1 \\
eGFR & $\left(\mathrm{mL} / \mathrm{min} / 1.73 \mathrm{~m}^{2}\right)$ & 56 \\
Sodium & $(\mathrm{mmol} / \mathrm{L})$ & 139 \\
Potassium & $(\mathrm{mmol} / \mathrm{L})$ & 2.8 \\
Chloride & $(\mathrm{mmol} / \mathrm{L})$ & 98 \\
Total protein & $(\mathrm{g} / \mathrm{dL})$ & 6.2 \\
Albumin & $(\mathrm{g} / \mathrm{dL})$ & 3.7 \\
Total cholesterol & $(\mathrm{mg} / \mathrm{dL})$ & 200 \\
HbA1c & $(\%)$ & 5.6 \\
Active renin concentration & $(\mathrm{pg} / \mathrm{mL})$ & 166 \\
Plasma aldosterone & $(\mathrm{pg} / \mathrm{mL})$ & 267 \\
Brain natriuretic peptide & $(\mathrm{pg} / \mathrm{mL})$ & 82 \\
Urine blood & & $(-)$ \\
Urine protein & $(\mathrm{g} / \mathrm{g} \mathrm{creatinine})$ & 4.6 \\
\hline
\end{tabular}

eGFR: estimated glomerular filtration rate nosed 2 years earlier and was presently being administered amlodipine $5 \mathrm{mg}$ per day with good blood pressure control. He smoked cigarettes (20 per day for 37 years) and did not have diabetes mellitus, hyperlipidemia, or obesity (body mass index: $22 \mathrm{~kg} / \mathrm{m}^{2}$ ). He presented with relatively sudden deterioration of blood pressure $(195 / 115 \mathrm{mmHg})$ with massive proteinuria (4.6 g/g creatinine) and polyuria. When an angiotensin II receptor blocker (ARB; olmesartan $20 \mathrm{mg}$ per day) was added to control his blood pressure, his serum creatinine level increased from 1.1 to $2.2 \mathrm{mg} / \mathrm{dL}$. After olmesartan was discontinued, the serum creatinine returned to 1.1 $\mathrm{mg} / \mathrm{dL}$. A laboratory examination showed hypokalemia and excessive elevation of the active renin and aldosterone concentrations (Table). Doppler ultrasound did not detect flow signals in the right renal artery, and enhanced computed tomography (CT) revealed total occlusion of the right renal artery with atrophy of the right kidney (Fig. 1A, B). The poleto-pole length of the right kidney had decreased to $69 \mathrm{~mm}$, while that of the left kidney was $111 \mathrm{~mm}$ (Fig. 1C). Technetium-99m-labelled diethylenetriamine-pentaacetic acid $\left({ }^{99 m}\right.$ Tc-DTPA) renography revealed no peak curve of the right kidney, suggesting no residual function in the affected kidney. On magnetic resonance imaging (MRI), diffusionweighted images demonstrated a high signal intensity with reduced apparent diffusion coefficients in the right kidney parenchyma (Fig. 1D, E), indicating restricted water diffusion due to severe ischemic changes (13-15). He presented with a regular pulse rate of 104 beats per minute and no evidence of thrombophilic disorders, aortic lesion, carotid artery stenosis, or ischemic heart disease. Based on these findings, we diagnosed him with renovascular hypertension
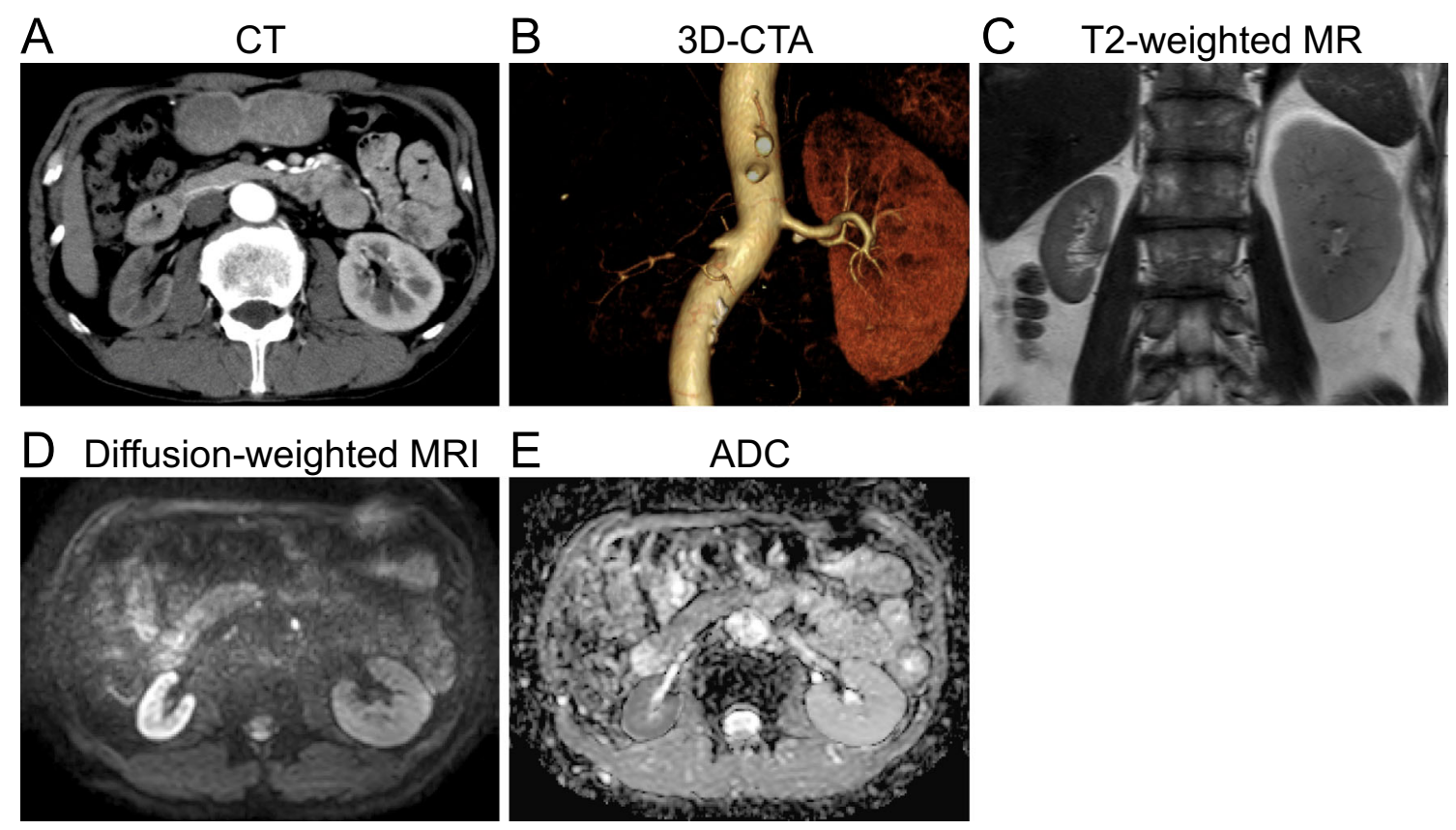

Figure 1. Total occlusion of the right renal artery, leading to renal atrophy. A: Enhanced computed tomography (CT) image. B: Three-dimensional CT angiography image. C: T2-weighted magnetic resonance imaging (MRI) scan. D: Diffusion-weighted MRI scan. E: Apparent diffusion coefficient (ADC) in diffusion-weighted MRI scan. 

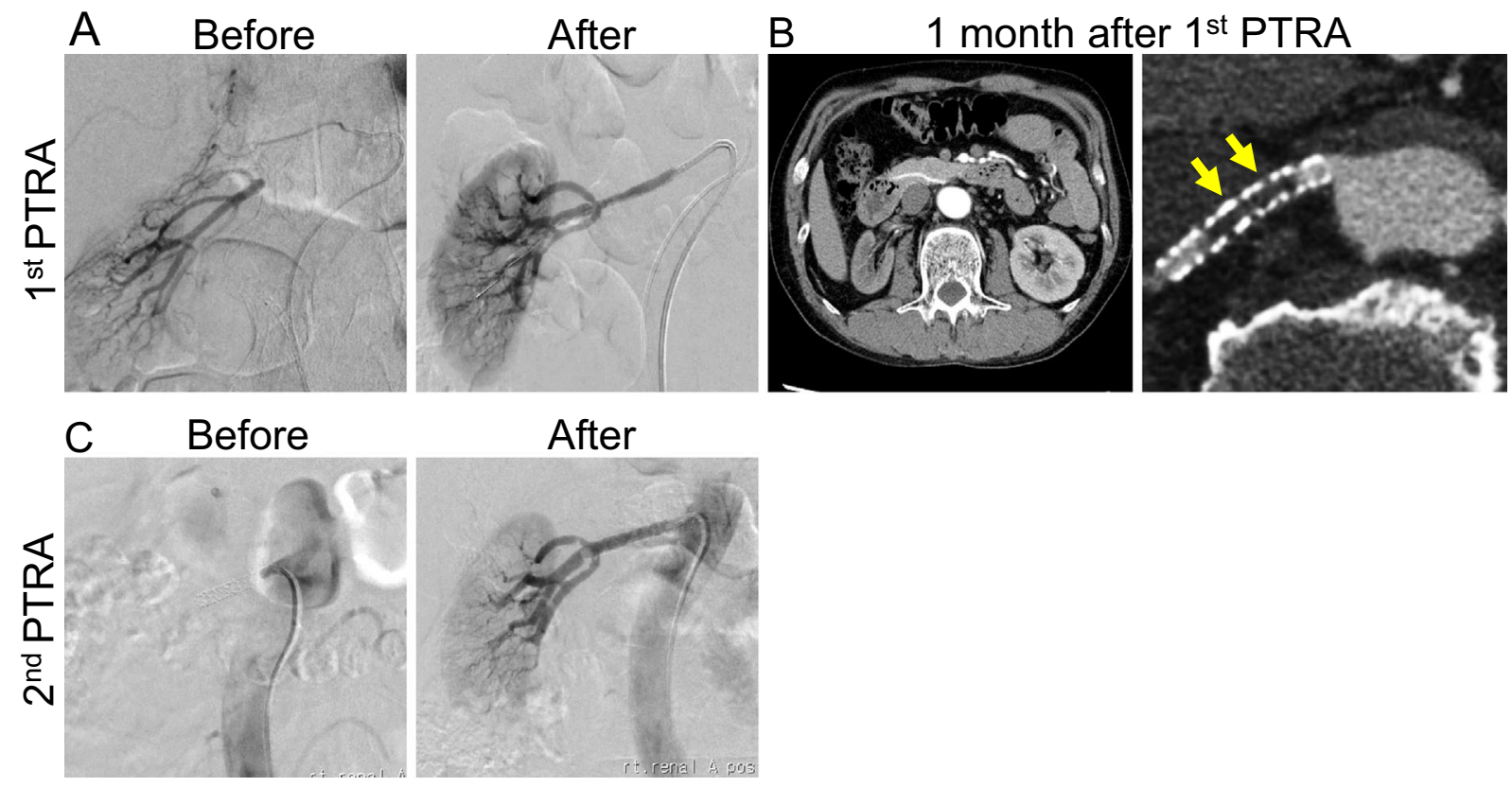

\begin{abstract}
Figure 2. Angioplasty for total occlusion in the renal artery. A: First percutaneous transluminal renal angioplasty (PTRA) procedure for the right renal artery. Angiography obtained by cannulating the adrenal artery providing collateral flow to the right kidney before PTRA and renal artery angiography after PTRA. B: Computed tomography one month after the first PTRA procedure showing thrombotic in-stent re-occlusion (arrows) C: Second PTRA procedure for the right renal artery.
\end{abstract}

secondary to atherosclerotic renal artery occlusion, leading to kidney atrophy.

Despite treatment with multiple antihypertensive medications (nifedipine $80 \mathrm{mg}$, doxazosin $8 \mathrm{mg}$, and clonidine 150 $\mu \mathrm{g}$ per day), the blood pressure remained uncontrollable at 160-200/100-130 mmHg. Thus, we decided to perform PTRA. At four months after the onset of deterioration of blood pressure, renal angiography showed occlusion of the right renal artery with collateral flow to the kidney from the adrenal artery (Fig. 2A). Balloon dilation and placement of 2 stents in the lesion revascularized the right kidney, although a systolic pressure gradient of $20 \mathrm{mmHg}$ remained across the treated lesion. He was started on aspirin $100 \mathrm{mg}$ the following day. After the procedure, his blood pressure and the plasma renin level decreased; however, they increased again one month after the treatment. CT showed thrombotic in-stent re-occlusion of the right renal artery (Fig. 2B). Thus, we performed endovascular balloon dilatation for the lesion again, and revascularization was achieved (Fig. 2C). After the second treatment, the plasma renin concentration dramatically decreased to $11 \mathrm{pg} / \mathrm{mL}$, and the blood pressure was controlled at $130 / 85 \mathrm{mmHg}$ with antihypertensive medication, including the ARB azilsartan $(20 \mathrm{mg}$ ) without a rapid decline in the renal function (Fig. 3). The urinary protein also decreased to $0.5 \mathrm{~g} / \mathrm{g}$ creatinine.

One month after the procedure, Doppler ultrasonography demonstrated a normalized intrarenal blood flow pattern and enlargement of the atrophic right kidney (length: $85 \mathrm{~mm}$ ), and ${ }^{99 \mathrm{~m}}$ Tc-DTPA scintigraphy showed partial recovery of the right kidney function (Fig. 4). After 6 months, the patient presented with favorable control of blood pressure (120/70 $\mathrm{mmHg}$ ) and a stable renal function (serum creatinine: 1.2 $\mathrm{mg} / \mathrm{dL}$ ), and no features of re-stenosis have been observed on follow-up evaluations by Doppler ultrasonography.

\section{Discussion}

In the present case, the timely resolution of the total occlusion of the renal artery by PTRA favorably achieved blood pressure control and functional and size recovery of the affected atrophic kidney in a patient with refractory hypertension. This suggests that PTRA can have a beneficial effect in select ARVD patients, even with an atrophic kidney and total occlusion of the renal artery, when the procedure is performed at an appropriate time. Thus, clinicians cannot exclude PTRA as a potential treatment for such patients and should carefully evaluate each individual case to decide whether or not performing PTRA promptly would provide any beneficial effect and thereby avoid missing the appropriate timing of PTRA.

Although the current expert consensus states that PTRA for ARVD with advanced atrophic kidney and/or chronic total occlusion is rarely appropriate (11), several case studies have reported a positive outcome in such patients (16-20). In the present case, the occlusive lesion appeared to progress during the time when hypertension had suddenly worsened. Angioplasty was performed four months after the onset of the deterioration of hypertension. In previous case reports as well, angioplasty achieved favorable blood pressure control in patients with advanced atrophic kidney who presented 


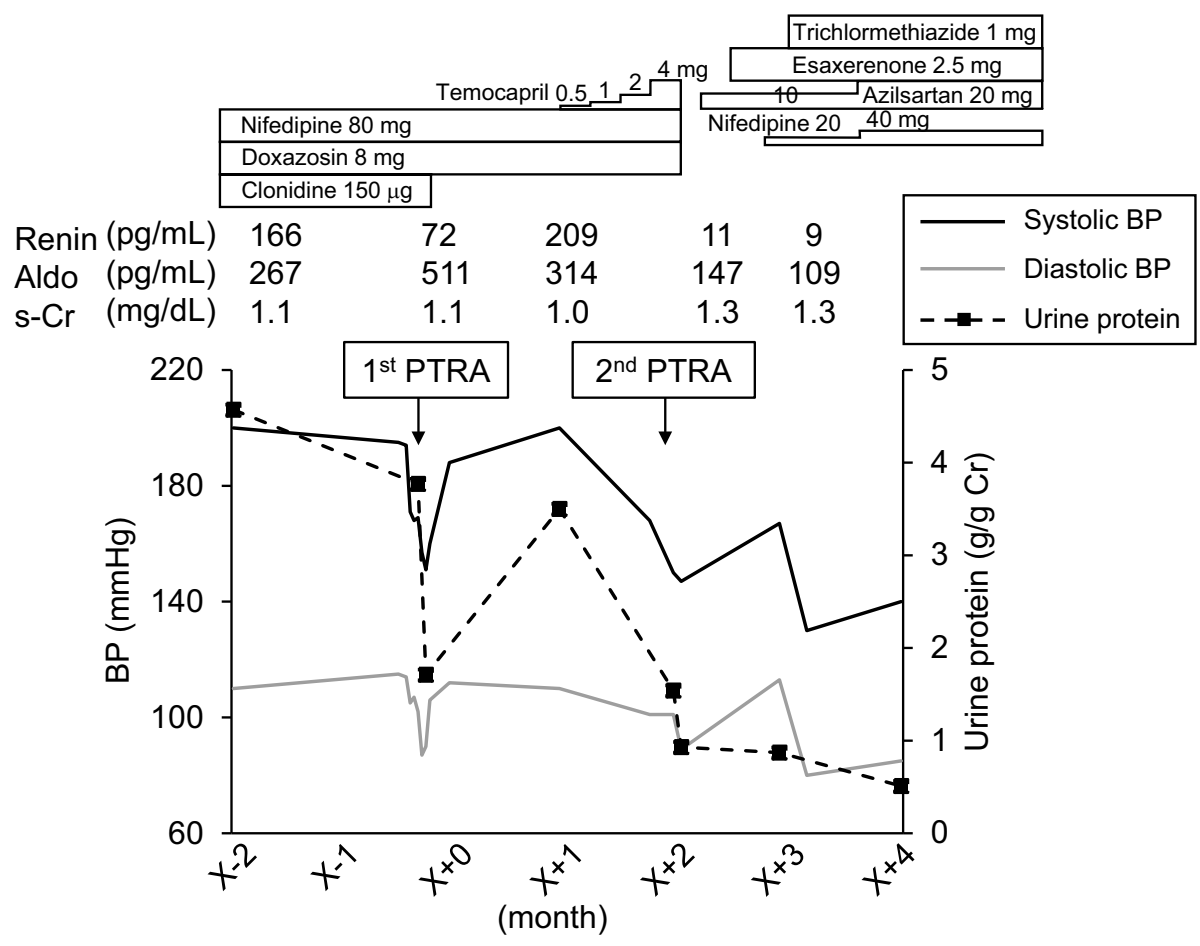

Figure 3. Clinical course. BP: blood pressure, PTRA: percutaneous transluminal renal angioplasty, Renin: active renin concentration, Aldo: aldosterone, s-Cr: serum creatinine
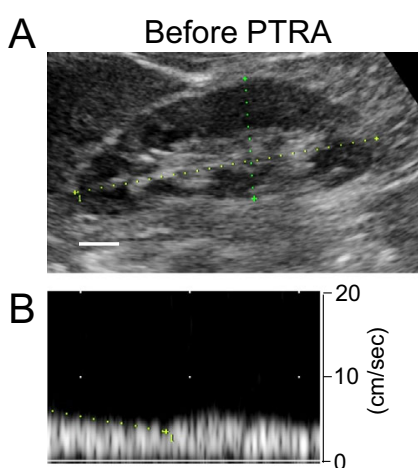

C

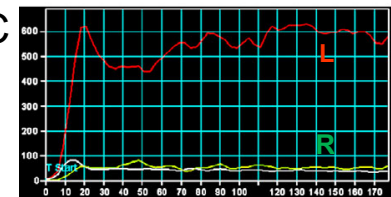

1 month after $2^{\text {nd }}$ PTRA
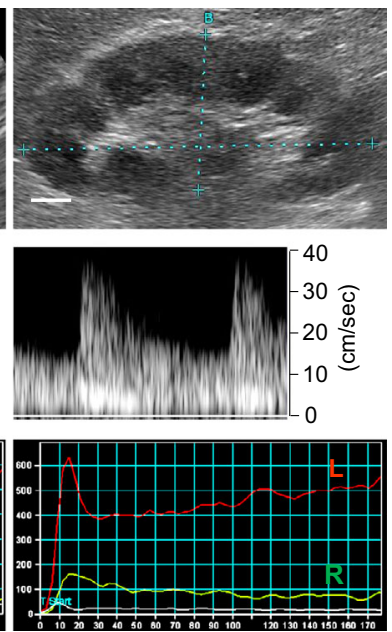

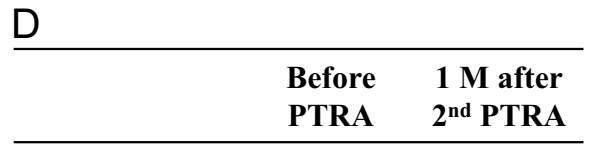

Echography

$\operatorname{PSV}(\mathrm{cm} / \mathrm{sec}) \quad 5$

$\operatorname{EDV}(\mathrm{cm} / \mathrm{sec}) \quad 4$

33

13

Resistive index $\quad 0.20$

0.59

AcT (msec)

188

38

Scintigraphy

Split function

(L:R, \%)

Figure 4. Kidney enlargement and improved intrarenal hemodynamics after the second PTRA procedure. A: The right kidney image on ultrasonography. Scale, $1 \mathrm{~cm}$. B: The intrarenal flow velocity pattern in the interlobar region in the right kidney. C: ${ }^{99 \mathrm{~m}}$ Tc-DTPA scintigraphy during the vascular phase. L: left kidney, R: right kidney. D: Parameters on ultrasonography and scintigraphy before and after PTRA. Pressure systolic velocity (PSV), endo diastolic velocity (EDV), resistive index, and acceleration time (AcT) were measured in the interlobar region in the right kidney.

with a sudden deterioration of blood pressure control, and PTRA was performed within six months after the onset of the episode $(18,19)$. When the sudden onset of hypertension or deterioration of blood pressure control leads to the diagnosis of ARVD, the involvement of a stenotic/occlusive lesion is very likely the cause of the blood pressure elevation. Thus, in patients with such features, angioplasty is theorized to improve hypertension. In addition, long-standing ischemia in the kidney would decrease the reversibility of renal parenchymal damage and reduce the beneficial effect of PTRA. Nevertheless, the role of these episodes in predicting the responsiveness of patients to angioplasty has not been evaluated in previous studies. The present case suggests that the timely resolution of the occlusive lesion by PTRA would exert a beneficial effect in patients who have had recent deterioration of blood pressure control (i.e. within the past six 
months), including those with atrophic kidneys.

Chronic total occlusion is defined as complete stenosis for more than three months (21). For the treatment of chronic total occlusion of the renal artery, angioplasty may functionally salvage the kidney with a preserved sub-segmental flow from the collateral arteries (22). Even without a blood supply from the main renal artery, the presence of collateral circulation can prevent renal infarction in the affected kidney, although the insufficient perfusion would cause renal ischemia and inappropriate renin secretion, leading to refractory hypertension. In such situations, revascularization can allow recovery of the function of the "hibernating" kidney and normalize the hyper-renin secretion because the kidney is not yet "dead" $(16,23)$. Indeed, although the present case showed almost no residual function in the affected kidney before PTRA, the function partially recovered, suggesting reversibility of the ischemic kidney that was only barely perfused by collateral supply. Previous reports showed that the recanalization of total renal artery occlusion in kidneys with collateral flow was feasible in the group of patients with refractory hypertension and high plasma rennin activity (22). Thus, when considering revascularization of the kidney with total renal artery occlusion, it is important to check for evidence of collateral flow to the kidney. To evaluate hibernating kidney, kidney function imaging has been performed (24). The present case indicates the beneficial use of diffusion-weighted MRI to detect kidney ischemia associated with renovascular disease, as we previously reported (13). However, further studies are warranted to more definitively elucidate the efficacy of diffusion-weighted MRI for evaluating the reversible hibernating kidney.

The present case showed in-stent re-occlusion after the first PTRA procedure. The residual stenosis indicated by the translesional pressure gradient may have contributed to the thrombus formation, although aspirin was prescribed to prevent re-occlusion after the treatment. In addition, a previous study on coronary artery disease showed that angioplasty for chronic total occlusion carries a higher risk of in-stent restenosis after treatment than that for non-occlusive stenosis (25).

The patient showed a slight increase in the serum creatinine concentration after PTRA, although it improved renal perfusion in the affected kidney. Regarding the pathophysiology of unilateral renal artery stenosis, while the poststenotic kidney has reduced perfusion, the contralateral kidney undergoes hyperperfusion and glomerular hyperfiltration associated with renin-angiotensin activation (1). The persistence of hyperperfusion results in parenchymal injury in the contralateral kidney. PTRA improves the renal reduced blood flow in the affected kidney and simultaneously normalizes compensatory hyperfiltration in the contralateral kidney (26). In addition, we administered a renin-angiotensin inhibitor in the present study, which also normalized the glomerular hyperfiltration (27). Thus, the increased creatinine concentration after the combination of PTRA and medication with renin-angiotensin inhibitors was attributed to the normalization of hyperfiltration in the contralateral kidney. Nevertheless, reducing the glomerular filtration via normalization of the activated renin-angiotensin system is thought to be beneficial for long-term renal protection (27).

In conclusion, PTRA is an effective option for treating ARVD patients who have refractory hypertension, even with an atrophic kidney and chronic total renal artery occlusion, especially in cases that meet specific criteria, such as recent blood pressure deterioration and the presence of collateral perfusion to the affected kidney. Clinicians should carefully evaluate patients by combining information from the clinical data, imaging modalities, and their timeline before deciding whether or not revascularization would be of any benefit.

The patient provided his informed consent.

\section{The authors state that they have no Conflict of Interest (COI).}

Yuri Sasaki and Eikan Mishima contributed equally to this work.

\section{References}

1. Herrmann SM, Textor SC. Current concepts in the treatment of renovascular hypertension. Am J Hypertens 31: 139-149, 2018.

2. Cooper CJ, Murphy TP, Cutlip DE, et al. Stenting and medical therapy for atherosclerotic renal-artery stenosis. $N$ Engl J Med 370: 13-22, 2014.

3. Investigators A, Wheatley $\mathrm{K}$, Ives $\mathrm{N}$, et al. Revascularization versus medical therapy for renal-artery stenosis. N Engl J Med 361: 1953-1962, 2009.

4. Japanese Society of Nephrology. Essential points from evidencebased clinical practice guidelines for chronic kidney disease 2018 . Clin Exp Nephrol 23: 1-15, 2019.

5. Whelton PK, Carey RM, Aronow WS, et al. 2017 ACC/AHA/ AAPA/ABC/ACPM/AGS/APhA/ASH/ASPC/NMA/PCNA Guideline for the prevention, detection, evaluation, and management of high blood pressure in adults: a report of the American College of Cardiology/American Heart Association Task Force on clinical practice guidelines. Hypertension 71: e13-e115, 2018.

6. Aboyans V, Ricco JB, Bartelink MEL, et al. 2017 ESC Guidelines on the diagnosis and treatment of peripheral arterial diseases, in collaboration with the European Society for Vascular Surgery (ESVS): document covering atherosclerotic disease of extracranial carotid and vertebral, mesenteric, renal, upper and lower extremity arteries Endorsed by: the European Stroke Organization (ESO) The Task Force for the Diagnosis and Treatment of Peripheral Arterial Diseases of the European Society of Cardiology (ESC) and of the European Society for Vascular Surgery (ESVS). Eur Heart J 39: 763-816, 2018.

7. Mishima E, Suzuki T, Ito S. Selection of patients for angioplasty for treatment of atherosclerotic renovascular disease: predicting responsive patients. Am J Hypertens 33: 391-401, 2020.

8. Iwashima Y, Fukuda T, Horio T, et al. Impact of percutaneous revascularization on left ventricular mass and its relationship to outcome in hypertensive patients with renal artery stenosis. Am J Hypertens 33: 570-580, 2020.

9. Fujihara M, Yokoi Y, Abe T, et al. Clinical outcome of renal artery stenting for hypertension and chronic kidney disease up to 12 months in the J-RAS Study - prospective, single-arm, multicenter clinical study. Circ J 79: 351-359, 2015.

10. Iwashima $Y$, Fukuda $T$, Horio $T$, et al. Association between renal 
function and outcomes after percutaneous transluminal renal angioplasty in hypertensive patients with renal artery stenosis. J Hypertens 36: 126-135, 2018.

11. Parikh SA, Shishehbor MH, Gray BH, White CJ, Jaff MR. SCAI expert consensus statement for renal artery stenting appropriate use. Catheter Cardiovasc Interv 84: 1163-1171, 2014.

12. Iwashima $Y$, Ishimitsu T. How should we define appropriate patients for percutaneous transluminal renal angioplasty treatment? Hypertens Res. Forthcoming.

13. Mishima E, Ota H, Suzuki T, et al. Apparent diffusion coefficient in the resolution of renal ischemia after angioplasty on diffusionweighted imaging: a case of renal artery stenosis caused by progressive thrombosis in residual chronic aortic dissection. Intern Med 59: 1173-1177, 2020.

14. Mishima E, Suzuki T, Seiji K, et al. Selective embolization therapy for intrarenal artery stenosis causing renovascular hypertension: efficacy and follow-up renal imaging. J Clin Hypertens (Greenwich) 19: 1028-1031, 2017.

15. Mishima E, Kikuchi K, Ota H, et al. Detection of segmental renal ischemia by diffusion-weighted magnetic resonance imaging: clinical utility for diagnosis of renovascular hypertension. J Clin Hypertens (Greenwich) 18: 364-365, 2016.

16. Manohar S, Hamadah A, Herrmann SM, Textor SC. Total renal artery occlusion: recovery of function after revascularization. Am J Kidney Dis 71: 748-753, 2018.

17. Yang M, Lin L, Niu G, et al. Successful endovascular treatment of chronic renal artery occlusion: a preliminary retrospective case series including 15 patients. Int Urol Nephrol 51: 285-291, 2019.

18. Maruyama K, Chinda J, Kabara M, et al. Successful percutaneous transluminal angioplasty for the treatment of renovascular hypertension with an atrophic kidney. Heart Vessels 30: 274-279, 2015.

19. Takamiya Y, Okamura K, Shirai K, Urata H. Successful depressor effect concomitant with complete normalization of high renin and aldosterone profile by percutaneous transluminal renal angioplasty in a patient with acute exacerbated heart failure with preserved ejection fraction. Am J Case Rep 20: 1684-1690, 2019.

20. Wakui H, Hosokawa Y, Oshikawa J, et al. Endovascular treatment of renal artery stenosis improves contralateral renal hypertrophy with nephrotic syndrome. CEN Case Rep 3: 53-55, 2014.

21. Galassi AR, Werner GS, Boukhris M, et al. Percutaneous recanalisation of chronic total occlusions: 2019 consensus document from the EuroCTO Club. EuroIntervention 15: 198-208, 2019.

22. Petrov I, Tasheva I, Garvanski I, Marzyanov M, Adam G. Recanalization and stenting of total occlusions of the renal arteries for blood pressure control in resistant to treatment hypertension. Cardiovasc Revasc Med. Forthcoming.

23. Schefft P, Novick AC, Stewart BH, Straffon RA. Renal revascularization in patients with total occlusion of the renal artery. J Urol 124: 184-186, 1980.

24. Chrysochou C, Green D, Ritchie J, Buckley DL, Kalra PA. Kidney volume to GFR ratio predicts functional improvement after revascularization in atheromatous renal artery stenosis. PLoS One 12: e $0177178,2017$.

25. Michael TT, Mogabgab O, Alomar M, et al. Long-term outcomes of successful chronic total occlusion percutaneous coronary interventions using the antegrade and retrograde approach. J Interv Cardiol 27: 465-471, 2014.

26. Iwasaki T, Mishima E, Suzuki T, et al. Kidney enlargement effect of angioplasty for nonatherosclerotic renovascular disease: reversibility of ischemic kidney. Hypertens Res. Forthcoming.

27. Helal I, Fick-Brosnahan GM, Reed-Gitomer B, Schrier RW. Glomerular hyperfiltration: definitions, mechanisms and clinical implications. Nat Rev Nephrol 8: 293-300, 2012.

The Internal Medicine is an Open Access journal distributed under the Creative Commons Attribution-NonCommercial-NoDerivatives 4.0 International License. To view the details of this license, please visit (https://creativecommons.org/licenses/ by-nc-nd/4.0/).

(C) 2021 The Japanese Society of Internal Medicine Intern Med 60: 287-292, 2021 